Bangladesh J. Bot. 49(4): 1003-1012, 2020 (December)

\title{
GENETIC DIVERSITY OF WALNUT (JUGLANS REGIA L.) SEEDLINGS THROUGH SSR MARKERS IN NORTH-WESTERN HIMALAYAN REGION OF JAMMU
}

\author{
Rafiq Ahmad Shah*, Parshant Bakshi ${ }^{1}$, Amit Jasrotia ${ }^{1}$, \\ DEEP JI BHAT ${ }^{1}$, RUCKU GUPTA ${ }^{1}$ AND MANISH BAKSHI ${ }^{1}$ \\ Central Institute of temperate Horticulture (ICAR) Old Airfield, \\ Rangreth-191132, Srinagar, Jammu \& Kashmir \\ Keywords: Cluster, Genetic diversity, SSR, Population, Walnut
}

\begin{abstract}
screening of 25 SSR markers, revealed 23 clear and consistent amplification profiles in the entire walnut germplasm set. A total of 54 alleles were amplified by SSR primers and the number of alleles range from 2 to 3 . The PIC value ranged from 0.36 to 0.68 . The dendrogram classified all genotypes into two main clusters with various degrees of subclustering. Estimated genetic dissimilarity coefficient ranged from 0.36 to 0.85. Through model-based cluster analysis all genotypes were grouped into 5 genetically distinct subpopulations. The expected heterozygosity at a given locus was found to range from 0.520 to 0.5477 . Similarly, population differentiation measurements (Fst) ranged from 0.2286 to 0.2909 . These findings would be helpful for decision making in future walnut breeding studies, germplasm management activities to maximize genetic diversity in walnut germplasm and may also prove useful in future for conducting association mapping in walnut for different traits.
\end{abstract}

\section{Introduction}

The Persian walnut is an important fruit tree in temperate regions of the world and it is grown for its commercial value. Jammu and Kashmir has genetically rich areas in walnut germplasm. Walnut being cross pollinated and growing under different agro-climatic conditions shows high variability in morpho-phenological traits in these areas. Observation of these traits has been the classical approach to study variation in walnut populations. But these characters usually vary with environment and evaluation of these traits requires growing the plants to full maturity prior identification. DNA markers enable characterization of genotypes independent of the influences of environmental growth conditions, physiological age of the plant and type of tissue being analyzed (Lavi et al. 1993). Among the DNA-based markers, microsatellites or SSRs allow a high level of resolution in genetic studies due to their high polymorphism, co-dominant inheritance, reproducibility, and easy detection by PCR (Gupta et al. 1996). There are many literature related to the use of microsatellites in the study of genetic relationships in walnut (Bai et al. 2010, Gunn et al. 2010). They exemplifies numerous applications in understanding the genetic structure of the genus Juglans (Victory et al. 2006) and also have been proven useful in the repository setting to examine potential redundancies and propagation errors within collections of walnut (Dangl et al. 2005). The collection, assessment and analysis of genetic diversity of walnut help in crop improvement programme. At the same time, it is necessary to develop better methods of characterization and evaluation of germplasm collections, to improve strategies for conservation and collection of germplasm and to increase the utilization of plant genetic resources. Keeping all the above facts in mind the main objective of this study was to examine the degree of genetic diversity and differentiation of walnut population collected from different areas of Jammu province and to understand the genetic relatedness among them with advanced method of SSR markers.

*Author for correspondence: <rafiq_masoodi@rediffmail.com>. ${ }^{1}$ Division of Fruit Science, Sher-e-Kashmir University of Agricultural Sciences \& Technology of Jammu, Faculty of Agriculture, Main Campus, Chatha, Jammu-180009. 


\section{Materials and Methods}

Young leaf samples from 84 genotypes/accessions were collected from the selected plants for DNA extraction. The genomic DNA isolation was carried out by following the standard method of Doyle and Doyle (1990), with slight modifications. Quality and quantity of genomic DNA was estimated through agarose gel electrophoresis and nanodrop (Thermo scientific USA). A set of 25 SSR marker primers selected from literature survey were used in the amplification of genomic DNA as listed in Table 1 along with primer sequences, annealing temperature and expected amplicon size. PCR was carried out in a $10 \mu \mathrm{l}$ reaction volume containing $1.25 \mu \mathrm{l}(25 \mathrm{mM} \mathrm{MgCl})$, $1 \mu \mathrm{l}$ dNTPs $(10 \mathrm{mM}), 1 \mu \mathrm{l}$ PCR buffer $(10 \mathrm{x}), 0.5 \mu \mathrm{l}(10 \mu \mathrm{M})$ forward primer, $0.5 \mu \mathrm{l}(10 \mu \mathrm{M})$ reverse primer primer, $2 \mu \mathrm{l}$ DNA template $(25-50 \mathrm{ng}), 0.15 \mu \mathrm{l}(5 \mathrm{units} / \mu \mathrm{l})$ DNA Taq polymerase and $3.6 \mu 1$ sterile water.

Table 1. List of selected SSR primers along with their primer sequence.

\begin{tabular}{|c|c|c|c|c|}
\hline Primer & $\begin{array}{l}\text { Forward primer } \\
\left(5^{\prime}-3^{\prime}\right)\end{array}$ & $\begin{array}{l}\text { Reverse primer } \\
\left(5^{\prime}-3{ }^{\prime}\right)\end{array}$ & $\begin{array}{l}\text { Annealing } \\
\text { Temp. }\left({ }^{\circ} \mathrm{C}\right)\end{array}$ & $\begin{array}{l}\text { Expected } \\
\text { amplicon } \\
\text { size (bp) }\end{array}$ \\
\hline WGA- 42 & F:GTGGGTTCGACCGTGAAC & R: AACTTTGCACCACATCCACA & 55 & $210-260$ \\
\hline WGA- 1 & F : ATTGGAAGGGAAGGGAAATG & R : CGCGCACATACGTAAATCAC & 56 & $180-210$ \\
\hline WGA-376 & F:GCCCTCAAAGTGATGAACGT & R:TCATCCATATTTACCCCTTTCG & 56 & $180-200$ \\
\hline WGA-71 & F: ACCCGAGAGATTTCTGGGAT & R:GGACCCAGCTCCTCTTCTCT & 57 & $150-165$ \\
\hline WGA-70 & F : TGTAATTGGGGAATGTTGCA & R : TGGGAGACACAATGATCGAA & 55 & $150-160$ \\
\hline WGA-276 & F: CTCACTTTCTCGGCTCTTCC & R: GGTCTTATGTGGGCAGTCGT & 60 & $140-160$ \\
\hline WGA-89 & F : ACCCATCTTTCACGTGTGTG & R : TGCCTAATTAGCAATTTCCA & 57 & $181-239$ \\
\hline WGA-72 & F : AAACCACCTAAAACCCTGCA & R : ACCCATCCATGATCTTCCAA & 55 & $150-170$ \\
\hline WGA-79 & F : CACTGTGGCACTGCTCATCT & R : TTCGAGCTCTGGACCACC & 55 & $204-220$ \\
\hline WGA-76 & F : AGGGCACTCCCTTATGAGGT & R : CAGTCTCATTCCCTTTTTCC & 58 & $228-254$ \\
\hline WGA-202 & F: CCCATCTACCGTTGCACTTT & R: GCTGGTGGTTCTATCATGGG & 62 & $240-300$ \\
\hline WGA-4 & F: TGTTGCATTGACCCACTTGT & R: TAAGCCAACATGGTATGCCA & 62 & $220-270$ \\
\hline WGA-9 & F: CATCAAAGCAAGCAATGGG & R: CCATTGGTCTGTGATTGGG & 56 & $230-290$ \\
\hline WGA69 & F: TTAGATTGCAAACCCACCCG & R: AGATGCACAGACCAACCCTC & 55 & $80-110$ \\
\hline WGA11 & F: CTCGACAGAAACAGCCACAA & R: GGAGTTGTGTGCAGTGCG & 55 & $205-225$ \\
\hline WGA-5 & F:CAGTTTGTCCCACACCTCCT & R:AACCCATGGTGAGAGAGTGAG & 62 & $185-210$ \\
\hline WGA118 & F:TGTGCTCTGATCTGCCTCCC & R: GGGTGGGTGAAAAGTAGCA & 60 & $190-260$ \\
\hline WGA045 & F: TCGTTACCACCAGCACAGAG & R: GACATAGCGAGGGGCTAGG & 60 & $250-315$ \\
\hline WGA331 & F:TCCCCCTGAAATCTTCTCCT & R:CGGTGGTGTAAGGCAAATG & 60 & $250-315$ \\
\hline WGA349 & F:GTGGCGAAAGTTTATTTTTTGC & R:ACAAATGCACAGCAGCAAAC & 60 & $250-290$ \\
\hline WGA321 & F:TCCAATCGAAACTCCAAAGG & R:GTCCAAAGACGATGATGGA & 56 & $240-265$ \\
\hline WGA 32 & F: CTCGGTAAGCCACACCAATT & R: ACGGGCAGTGTATGCATGTA & 58 & $163-235$ \\
\hline WGA332 & F:ACGTCGTTCTGCACTCCTCT & R:GCCACAGGAACGAGTGCT & 57 & $230-300$ \\
\hline WGA 27 & F: AACCTCACGCCTTGATG & R: TGC TCA GGC TCC ACT TCC & 57 & $225-260$ \\
\hline WGA225 & F:AATCCCTCTCCTGGGCAG & R:TGTTCCACTGACCACTTCCA & 58 & $200-210$ \\
\hline
\end{tabular}


The PCR was run in thermal cycler machine (Peq STAR, German manufactured) for DNA amplification. PCR steps consist of an initial denaturation step of $5 \mathrm{~min}$, followed by a loop of 35 cycles each consisting of denaturation (at $94^{\circ} \mathrm{C}$ for $1 \mathrm{~min}$ ), annealing (at $53-60^{\circ} \mathrm{C}$ for $1 \mathrm{~min}$ and $40 \mathrm{sec}$ ), elongation (at $72^{\circ} \mathrm{C}$ for $40 \mathrm{sec}$ ) and the final extension was performed (at $72^{\circ} \mathrm{C}$ for $5 \mathrm{~min}$ ). Amplified DNA fragments were resolved in horizontal electrophoresis by using 3\% agarose gels and visualized in gel documentation system (Bio-Rad,USA). The DNA bands were scored on the basis of relative mobility in gel, the allele difference was determined according to their fragment size (bp) corresponding to the $100 \mathrm{bp}$ standard marker (Sigma Aldrich, U.S.A). The molecular data obtained were used to study in computer software program DARwin 5 (Perrier and Jacquemoud-Collet 2006) for dendrogram construction. In order to check the informativeness and discriminatory power of SSR markers used in this study, certain parameters like polymorphism information content and alleles per locus were calculated. Model-based cluster analysis was performed to infer the genetic structure and to define the number of clusters (gene pools) in the data set using the software STRUCTURE v.2.3.4 (Pritchard et al. 2000). The number of presumed population (K) was set from 1 to 10 , and analysis was repeated two times. For each run, burn-in and iterations were set to $1,00,000$ and 2,00,000, respectively and a model without admixture and correlated allel frequencies was used. The optimum value of $\mathrm{K}$ was determined by calculating the $1 \mathrm{~K}$ value to estimate the most likely number of groups (Evanno et al. 2005). The results were processed with the software STRUCTURE HARVESTER v.0.6.1 (Earl and Von Holdt 2012) to obtain the most likely $\mathrm{K}$ value. Within a group, genotypes with affiliation probabilities (inferred ancestry) $\geq 80 \%$ were assigned to a distinct group and those with $<80 \%$ were treated as "admixture". The expected heterozygosity (gene diversity) and population differentiation (Fst) between individual in a sub-population were also worked out using STRUCTURE programme.

\section{Results and Discussion}

A total of 54 alleles were amplified by 23 polymorphic SSR loci and the number of alleles ranged from 2 to 3 with an average of 2.34 alleles per locus (Table 2). These results are similar to previous results reported by Ahmed et al. (2012) who screened 13 SSR primers in walnut and observed an average of 2 polymorphic loci per primer. Polymorphism among individual genotypes is observed due to variation in DNA sequences present in their chromosomes. Primers WGA-05, WGA-01, WGA-76, WGA-09 and WGA-045 were more efficient to discriminate the genotypes than other primers. Higher polymorphic bands of primers indicate their efficiency to study genetic diversity and discrimination of genotypes (Pradhan et al. 2004). The PIC values ranged from 0.36 (WGA-321) to 0.68 (WGA-5) with an average value of 0.54 across all genotypes. These results are concurrent with earlier findings of Ahmed et al. (2012) and Najafi et al. (2014). The dendrogram (Fig. 1) classified all genotypes into two main clusters, cluster I and cluster II with various degrees of sub-clustering. The cluster I consisted of 78 genotypes. Cluster I was further divided into two sub-clusters IA and IB and contain 29 and 49 genotypes. Sub-cluster IB is further divided into two subgroups containing 39 and 10 genotypes, respectively. Cluster II contained six genotypes viz., JWSM-51, JMSM-50, JMSM-52, JWSM-84, JWSM-55 and JWSD-44. Although SSR markers were able to discriminate all the genotypes effectively, but there was no relationship between the spatial and genetic proximity of the germplasm apart from a few genotypes which were collected from the same location and genotypes collected from particular area showed diverse clustering, which may be due to out-crossing nature of walnut (Germain 1997). Estimated genetic dissimilarity coefficient ranged from 0.36 (JWSM-33 and JWSM-35) to 0.85 (JWSP-6 and JWSG-43). Wang et al. (2007) studied genetic diversity among five representatives of Juglans regia and four representatives of Juglans sigilata and found that the genetic distance varied from 0.049 to 0.84 and nine populations got grouped into two clusters. On the basis of the cluster 
analysis, it may be concluded that the walnut germplasm used for present study have a wide genetic base owing to the cross pollination behaviour of walnut. The dendrogram generated using SSR markers along with the phenotypic information will be useful for choosing the diverse parents for further walnut breeding programs. The present findings further strengthened previous reports that SSR markers can be used effectively to estimate genetic differences among genotypes and are in agreement with the results reported by Shah et al. (2016) in walnut in which the studied genotypes were discriminated according to the gene pool level by UPGMA based cluster analysis. Moreover, recent studies revealed the potential of SSRs in providing intraspecific diversity within genus.

Table 2. Genetic characterization of 23 microsatellite loci for 84 walnut (Juglans regia $\mathrm{L}$.) genotypes native to Jammu province.

\begin{tabular}{clcc}
\hline S. No. & Primer & PIC value & Allele/locus \\
\hline 1 & WGA- 42 & 0.56 & 2 \\
2 & WGA- 01 & 0.66 & 2 \\
3 & WGA-376 & 0.65 & 2 \\
4 & WGA-71 & 0.54 & 2 \\
5 & WGA-70 & 0.63 & 2 \\
6 & WGA-276 & 0.51 & 2 \\
7 & WGA-89 & 0.57 & 2 \\
8 & WGA-72 & 0.54 & 3 \\
9 & WGA-79 & 0.55 & 2 \\
10 & WGA-76 & 0.60 & 2 \\
11 & WGA-202 & 0.37 & 2 \\
12 & WGA-04 & 0.54 & 3 \\
13 & WGA-09 & 0.50 & 3 \\
14 & WGA-69 & 0.65 & 2 \\
15 & WGA-11 & 0.58 & 3 \\
16 & WGA-05 & 0.68 & 2 \\
17 & WGA-118 & 0.40 & 3 \\
18 & WGA-045 & 0.61 & 3 \\
19 & WGA-331 & 0.44 & 2 \\
20 & WGA-349 & 0.54 & 3 \\
21 & WGA-321 & 0.36 & 2 \\
22 & WGA-32 & 0.56 & 2 \\
23 & WGA-332 & 0.54 & 2 \\
\hline
\end{tabular}

Model-based cluster analysis grouped 84 walnut genotypes into 5 genetically distinct subpopulations (K5), having maximum natural log probability (-7555.9), which is proportional to the posterior probability. For the admixture and correlated frequency model, the log-likelihood value $\mathrm{L}(\mathrm{K})$ as a function of $\mathrm{K}$ (number of clusters) averaged over two replicates increased almost linearly from $\mathrm{K}=1(-8477.6)$ up to $\mathrm{K}=5(-7,555.2)$. The approach of Evanno et al. (2005) strongly supported $\mathrm{K}=5$ as the most likely number of clusters (Fig. 2), because the highest second order of change of the log-likelihood of the data $(\Delta K)$, as a function of $K$, was detected at $K=5$. In addition to assignments of some individuals to a population, some degree of admixtures was also observed (Table 3). Among 84 individuals, 9, 6, 7, 9, 8 individuals belong to population 1, 2, 
$3,4,5$, respectively and rest of the 45 individuals belong to different populations with varied proportion of admixtures (Fig. 3). The overall proportion of membership of populations without admixtures was $21.05,15.78,13.10,23.68$ and $21.05 \%$ for population $1,2,3,4$ and 5 , respectively. The present results agree with the results reported by Shah et al. (2016) in walnut. The expected heterozygosity, at a given locus ranges from 0.5201 in fourth sub-population to 0.5477 in third sub-population with an average of 0.534. Similarly, population differentiation

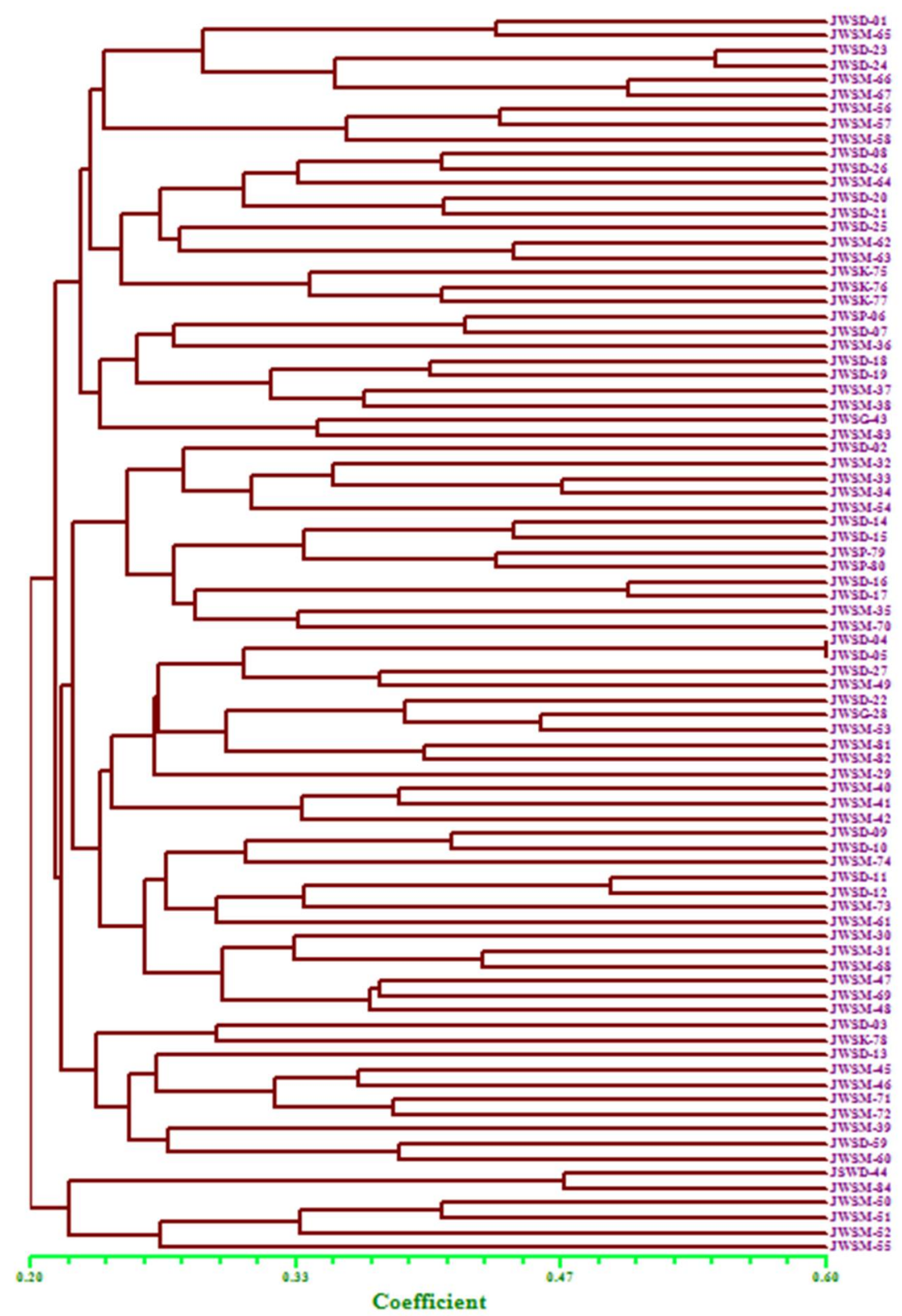

Fig. 1. UPGMA dendrogram showing clustering pattern of 84 walnut genotypes. 
1008

Table 3. Assignment of sub-populations (K) to the individuals based on probability.

\begin{tabular}{|c|c|c|c|c|c|c|c|}
\hline Code & Genotype & K-1 & K-2 & $\mathrm{K}-3$ & K-4 & K-5 & Sub-population \\
\hline 1 & JWSD-01 & 0.475 & 0.013 & 0.269 & 0.218 & 0.025 & Admixture of $1,3 \& 4$ \\
\hline 2 & JWSD-02 & 0.716 & 0.024 & 0.054 & 0.062 & 0.144 & Admixture of $1 \& 5$ \\
\hline 3 & JWSD-03 & 0.675 & 0.008 & 0.015 & 0.228 & 0.075 & Admixture of $1 \& 4$ \\
\hline 4 & JWSD-04 & 0.922 & 0.020 & 0.006 & 0.013 & 0.039 & 1 \\
\hline 5 & JWSD-05 & 0.946 & 0.006 & 0.005 & 0.013 & 0.029 & 1 \\
\hline 6 & JWSP-06 & 0.304 & 0.010 & 0.580 & 0.084 & 0.022 & Admixture of $1, \& 3$ \\
\hline 7 & JWSD-07 & 0.013 & 0.011 & 0.481 & 0.313 & 0.182 & Admixture of $3,4 \& 5$ \\
\hline 8 & JWSD-08 & 0.014 & 0.010 & 0.074 & 0.870 & 0.032 & 4 \\
\hline 9 & JWSD-09 & 0.013 & 0.008 & 0.006 & 0.958 & 0.016 & 4 \\
\hline 10 & JWSD-10 & 0.046 & 0.030 & 0.081 & 0.802 & 0.042 & 4 \\
\hline 11 & JWSD-11 & 0.008 & 0.020 & 0.021 & 0.302 & 0.648 & Admixture of $4 \& 5$ \\
\hline 12 & JWSD-12 & 0.019 & 0.028 & 0.014 & 0.068 & 0.872 & 5 \\
\hline 13 & JWSD-13 & 0.110 & 0.149 & 0.013 & 0.168 & 0.561 & Admixture of $1,2,4 \& 5$ \\
\hline 14 & JWSD-14 & 0.531 & 0.419 & 0.010 & 0.006 & 0.034 & Admixture of $1 \& 2$ \\
\hline 15 & JWSD-15 & 0.183 & 0.266 & 0.021 & 0.012 & 0.517 & Admixture of $1,2 \& 5$ \\
\hline 16 & JWSD-16 & 0.027 & 0.008 & 0.009 & 0.007 & 0.949 & 5 \\
\hline 17 & JWSD-17 & 0.013 & 0.011 & 0.021 & 0.008 & 0.947 & 5 \\
\hline 18 & JWSD-18 & 0.008 & 0.019 & 0.602 & 0.336 & 0.035 & Admixture of $3 \& 4$ \\
\hline 19 & JWSD-19 & 0.018 & 0.040 & 0.609 & 0.308 & 0.025 & Admixture of $3 \& 4$ \\
\hline 20 & JWSD-20 & 0.017 & 0.033 & 0.631 & 0.242 & 0.077 & Admixture of $3 \& 4$ \\
\hline 21 & JWSD-21 & 0.144 & 0.288 & 0.013 & 0.502 & 0.053 & Admixture of $2 \& 4$ \\
\hline 22 & JWSD-22 & 0.517 & 0.309 & 0.020 & 0.148 & 0.006 & Admixture of $1,2 \& 4$ \\
\hline 23 & JWSD-23 & 0.115 & 0.006 & 0.004 & 0.870 & 0.005 & 4 \\
\hline 24 & JWSD-24 & 0.069 & 0.009 & 0.010 & 0.903 & 0.009 & 4 \\
\hline 25 & JWSD-25 & 0.006 & 0.012 & 0.302 & 0.645 & 0.033 & Admixture of $3 \& 4$ \\
\hline 26 & JWSD-26 & 0.127 & 0.021 & 0.138 & 0.668 & 0.046 & Admixture of $3 \& 4$ \\
\hline 27 & JWSD-27 & 0.602 & 0.357 & 0.018 & 0.012 & 0.011 & Admixture of $1 \& 2$ \\
\hline 29 & JWSM-29 & 0.711 & 0.116 & 0.065 & 0.088 & 0.020 & 1 \\
\hline 30 & JWSM-30 & 0.669 & 0.181 & 0.066 & 0.048 & 0.036 & Admixture of $1 \& 2$ \\
\hline 31 & JWSM-31 & 0.312 & 0.067 & 0.008 & 0.024 & 0.589 & Admixture of $1 \& 5$ \\
\hline 32 & JWSM-32 & 0.409 & 0.008 & 0.010 & 0.010 & 0.563 & Admixture of $1 \& 5$ \\
\hline 33 & JWSM-33 & 0.838 & 0.019 & 0.076 & 0.014 & 0.054 & 1 \\
\hline 34 & JWSM-34 & 0.944 & 0.010 & 0.012 & 0.008 & 0.026 & 1 \\
\hline 35 & JWSM-35 & 0.023 & 0.013 & 0.006 & 0.064 & 0.894 & 5 \\
\hline 36 & JWSM-36 & 0.214 & 0.031 & 0.253 & 0.252 & 0.250 & Admixture of $3,4 \& 5$ \\
\hline 37 & JWSM-37 & 0.092 & 0.010 & 0.855 & 0.036 & 0.007 & 3 \\
\hline 38 & JWSM-38 & 0.012 & 0.043 & 0.818 & 0.060 & 0.066 & 3 \\
\hline 39 & JWSM-39 & 0.015 & 0.054 & 0.494 & 0.014 & 0.422 & Admixture of $3 \& 5$ \\
\hline 40 & JWSM-40 & 0.360 & 0.367 & 0.071 & 0.191 & 0.011 & Admixture of $1 \& 2$ \\
\hline 41 & JWSM-41 & 0.382 & 0.352 & 0.177 & 0.073 & 0.017 & Admixture of $1 \& 2$ \\
\hline 42 & JWSM-42 & 0.016 & 0.223 & 0.694 & 0.061 & 0.006 & 3 \\
\hline
\end{tabular}


(Contd.)

\begin{tabular}{|c|c|c|c|c|c|c|c|}
\hline 43 & JWSG-43 & 0.010 & 0.737 & 0.040 & 0.157 & 0.056 & 2 \\
\hline 44 & JWSD-44 & 0.012 & 0.037 & 0.751 & 0.007 & 0.194 & 3 \\
\hline 45 & JWSM-45 & 0.006 & 0.052 & 0.097 & 0.046 & 0.800 & 5 \\
\hline 46 & JWSM-46 & 0.052 & 0.040 & 0.018 & 0.023 & 0.868 & 5 \\
\hline 47 & JWSM-47 & 0.006 & 0.934 & 0.027 & 0.015 & 0.017 & 2 \\
\hline 48 & JWSM-48 & 0.007 & 0.810 & 0.009 & 0.015 & 0.159 & 2 \\
\hline 49 & JWSM-49 & 0.219 & 0.577 & 0.020 & 0.006 & 0.178 & Admixture of $1,2 \& 5$ \\
\hline 50 & JWSM-50 & 0.141 & 0.017 & 0.686 & 0.123 & 0.033 & Admixture of $1 \& 4$ \\
\hline 51 & JWSM-51 & 0.016 & 0.009 & 0.443 & 0.368 & 0.164 & Admixture of $2 \& 4$ \\
\hline 52 & JWSM-52 & 0.315 & 0.020 & 0.375 & 0.125 & 0.164 & Admixture of $1,3,4 \& 5$ \\
\hline 53 & JWSM-53 & 0.912 & 0.050 & 0.007 & 0.018 & 0.013 & 1 \\
\hline 54 & JWSM-54 & 0.701 & 0.018 & 0.224 & 0.031 & 0.027 & 1 \\
\hline 55 & JWSM-55 & 0.361 & 0.055 & 0.366 & 0.147 & 0.071 & Admixture of $1,3 \& 4$ \\
\hline 56 & JWSM-56 & 0.009 & 0.018 & 0.463 & 0.494 & 0.016 & Admixture of $1 \& 4$ \\
\hline 57 & JWSM-57 & 0.057 & 0.132 & 0.048 & 0.756 & 0.007 & 4 \\
\hline 58 & JWSM-58 & 0.043 & 0.295 & 0.034 & 0.620 & 0.008 & Admixture of $2 \& 4$ \\
\hline 59 & JWSD-59 & 0.012 & 0.883 & 0.027 & 0.007 & 0.071 & 2 \\
\hline 60 & JWSM-60 & 0.026 & 0.494 & 0.016 & 0.015 & 0.449 & Admixture of $2 \& 5$ \\
\hline 61 & JWSM-61 & 0.056 & 0.141 & 0.059 & 0.707 & 0.037 & 4 \\
\hline 62 & JWSM-62 & 0.038 & 0.076 & 0.676 & 0.202 & 0.007 & Admixture of $3 \& 4$ \\
\hline 63 & JWSM-63 & 0.008 & 0.036 & 0.710 & 0.230 & 0.017 & 3 \\
\hline 64 & JWSM-64 & 0.014 & 0.020 & 0.855 & 0.019 & 0.092 & 3 \\
\hline 65 & JWSM-65 & 0.305 & 0.020 & 0.502 & 0.166 & 0.006 & Admixture of $1,3 \& 4$ \\
\hline 66 & JWSM-66 & 0.036 & 0.009 & 0.071 & 0.843 & 0.041 & 4 \\
\hline 67 & JWSM-67 & 0.011 & 0.030 & 0.012 & 0.770 & 0.177 & 4 \\
\hline 68 & JWSM-68 & 0.007 & 0.938 & 0.008 & 0.024 & 0.023 & 2 \\
\hline 69 & JWSM-69 & 0.059 & 0.876 & 0.007 & 0.030 & 0.027 & 2 \\
\hline 70 & JWSM-70 & 0.273 & 0.085 & 0.016 & 0.098 & 0.527 & Admixture of $1 \& 5$ \\
\hline 71 & JWSM-71 & 0.073 & 0.018 & 0.083 & 0.016 & 0.810 & 5 \\
\hline 72 & JWSM-72 & 0.008 & 0.013 & 0.010 & 0.008 & 0.960 & 5 \\
\hline 73 & JWSM-73 & 0.009 & 0.009 & 0.013 & 0.295 & 0.674 & Admixture of $4 \& 5$ \\
\hline 74 & JWSM-74 & 0.044 & 0.039 & 0.010 & 0.400 & 0.508 & Admixture of $4 \& 5$ \\
\hline 75 & JWSK -75 & 0.018 & 0.431 & 0.061 & 0.213 & 0.277 & Admixture of $4 \& 5$ \\
\hline 76 & JWSK -76 & 0.013 & 0.749 & 0.161 & 0.009 & 0.069 & Admixture of $2 \& 3$ \\
\hline 77 & JWSK -77 & 0.005 & 0.648 & 0.270 & 0.019 & 0.059 & Admixture of $2 \& 3$ \\
\hline 78 & JWSK -78 & 0.008 & 0.418 & 0.047 & 0.258 & 0.269 & Admixture of $2,4 \& 5$ \\
\hline 79 & JWSP -79 & 0.019 & 0.762 & 0.017 & 0.017 & 0.185 & Admixture of $2 \& 5$ \\
\hline 80 & JWSP -80 & 0.080 & 0.734 & 0.048 & 0.014 & 0.124 & Admixture of $2 \& 5$ \\
\hline 81 & JWSM-81 & 0.183 & 0.391 & 0.315 & 0.015 & 0.097 & Admixture of $2 \& 3$ \\
\hline 82 & JWSM-82 & 0.017 & 0.378 & 0.334 & 0.228 & 0.042 & Admixture of $2,3 \& 4$ \\
\hline 83 & JWSM-83 & 0.029 & 0.462 & 0.328 & 0.164 & 0.017 & Admixture of $2 \& 3$ \\
\hline 84 & JWSM-84 & 0.013 & 0.011 & 0.967 & 0.004 & 0.005 & 3 \\
\hline
\end{tabular}


measurement (Fst) ranged from 0.2286 (in fifth sub-population) to 0.2909 (in fourth subpopulation), with an average of 0.259 (Table 4). Similar type of results was observed in Sorrento walnut using SSR primers by Foroni et al. (2007). Allele frequency divergence among populations computed using point estimates of $\mathrm{p}$ varies from 0.1155 between population 1 and 2 to 0.1530 between population 2 and 3 with an average of 0.2587 between all the five populations. The information gained through population structure analysis will prove useful in future for conducting association mapping in walnut for a variety of traits.

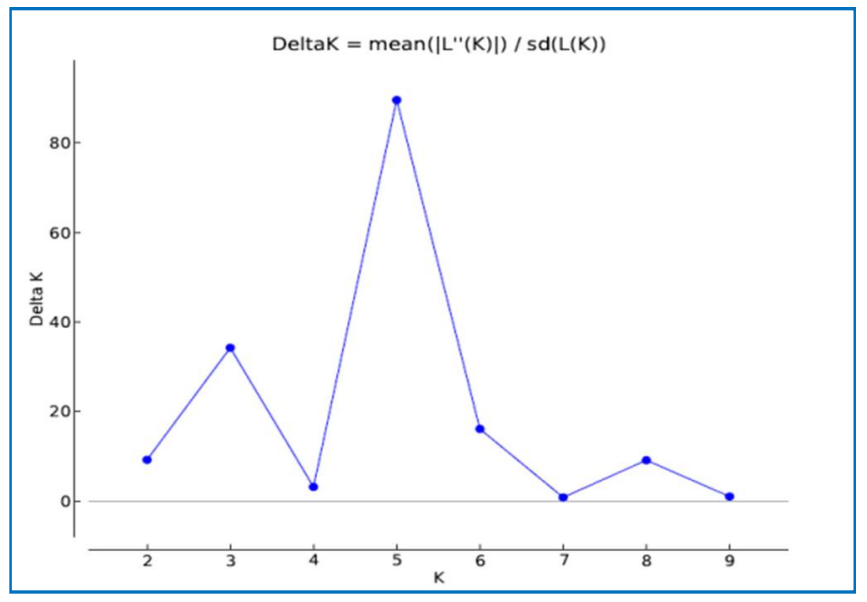

Fig. 2. Second order of change of the log likelihood of the data (Delta K) as a function of $\mathrm{K}$, calculated over two replications.

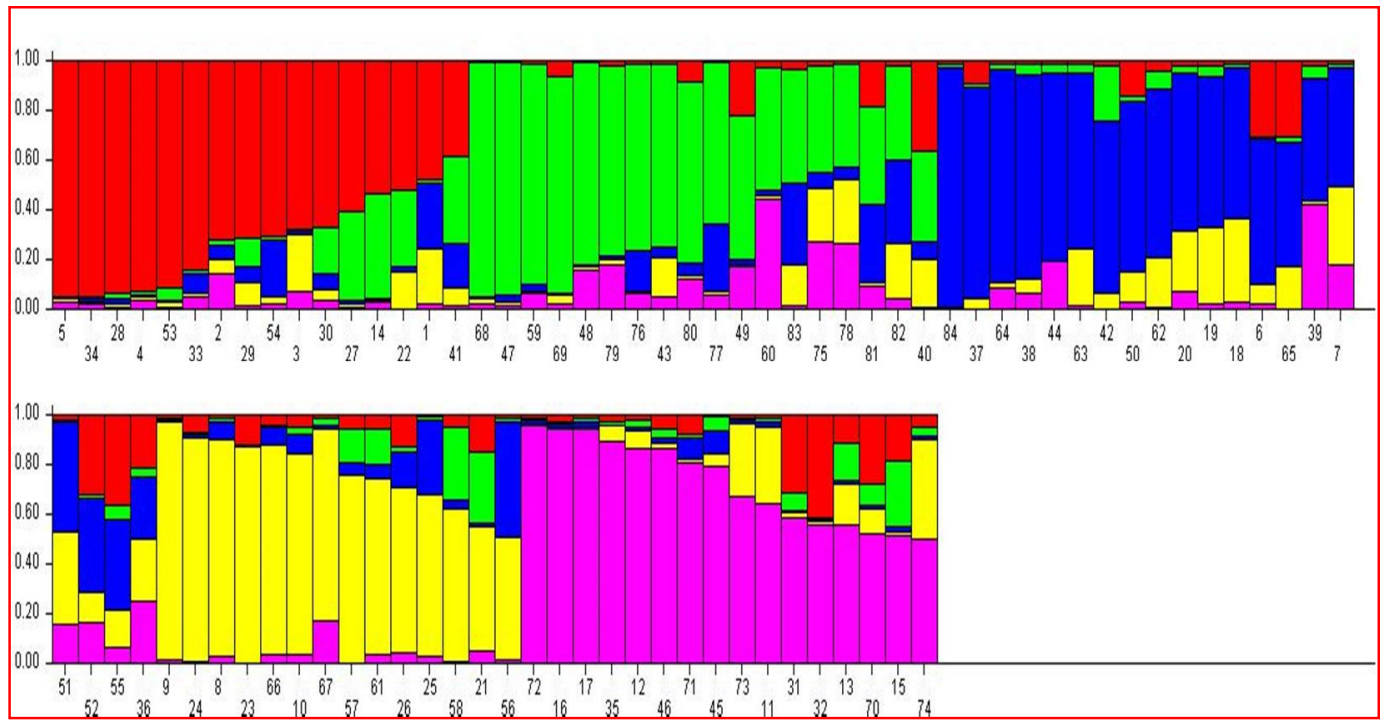

Fig. 3. Graphical representation of population structure in the walnut selections/genotypes. Each individual is represented by a vertical line and different colors in the same line indicate the individual's estimated 
membership percentage in $\mathrm{K}$ clusters (admixture proportion or $\mathrm{Q}$ value): red $=$ cluster 1 , light green $=$ cluster 2 , blue $=3$ yellow $=$ cluster 4 and purple $=5$.

Table 4. Heterozygosity and Fst value calculated for 5 walnut sub-populations.

\begin{tabular}{cccc}
\hline Sl. No. & Sub-population & Expected heterozygosity & F value \\
\hline 01 & 1 & 0.5390 & 0.2327 \\
02 & 2 & 0.5305 & 0.2742 \\
03 & 3 & 0.5477 & 0.2719 \\
04 & 4 & 0.5201 & 0.2909 \\
05 & 5 & 0.5335 & 0.2286 \\
Average & & 0.534 & 0.259 \\
\hline
\end{tabular}

From this study it may be concluded that SSR and STRUCTURE analysis has separated the accessions into three and 5 major genetically different groups, respectively with no spatial differentiation corresponding to an area and also indicated that the walnut genotypes are genetically different with various degrees of admixtures. These findings would provide valuable information for decision making in future walnut breeding studies as well as germplasm management activities to maximize genetic diversity in walnut germplasm. The molecular approach generates an unbiased picture of diversity than morphological and biochemical approaches. However, molecular diversity studies should be seen as equilibrating the traditional approach because most desirable traits are the results of interaction among expressed genes.

\section{References}

Ahmed N, Mir JI, Mir RR, Rather NA, Rashid R, Wani SH, Shafi W, Mir H and Sheikh MA 2012. SSR and RAPD analysis of genetic diversity in walnut (Juglans regia L.) genotypes from Jammu and Kashmir, India. Physiol. Mol. Biol. Plants. 18: 149-160.

Bai WN, Liao WJ and Zhang DY 2010. Nuclear and chloroplast DNA phylogeography reveal two refuge areas with asymmetrical gene flow in a temperate walnut tree from East Asia. New Phytol. 188: 892901.

Dangl GS, Woeste MK, Aradhya MK, Koehmstedt A and Simon C 2005. Characterization of 14 microsatellite markers for genetic analysis and cultivar identification of walnut. J. Am. Soc. Hort. Sci. 130: $348-354$.

Doyle JJ and Doyle JL 1990. Isolation of plant DNA from fresh tissue. Focus. 13-15.

Earl DA and VonHoldt BM 2012. Structure harvester: A website and program for visualizing structure output and implementing the Evanno method. Conserv. Genet. Resour. 4: 359-361.

Evanno G, Regnaut S and Goudet J 2005. Detecting the number of clusters of individuals using the software STRUCTURE, a simulation study. Mol. Ecol. 14: 2611-2620.

Foroni I, Woeste K, Monti LM and Rao R 2007. Identification of 'Sorrento' walnut using simple sequence repeats (SSRs). Genet. Resour. Crop Evol. 54: 1081-1094.

Germain E 1997. Genetic improvement of the persian walnut (Juglans regia L.). Acta Hortic. 442: 21-31.

Gunn BF, Aradhya M, Salick JM, Miller AJ, Yongping Y, Lin L and Xian H 2010. Genetic variation in walnuts (Juglans regia and J. sigillata; Juglandaceae) species distinctions, human impacts, and the conservation of agro biodiversity in Yunnan, China. Am. J. Bot. 97: 660-671.

Gupta P K, Balyan H S, Sharma PC and Ramesh B 1996. Microsatellites in plants, a new class of molecular markers. Curr. Sci. 70: 45-54.

Lavi U, Cregan PB, and Hillel J. 1993. Application of DNA markers for identification and breeding of fruit trees. Plant Breeding Rev. 16: (In press) Incomplete ?????? 7:195-226 
Najafi F, Mardi M, Fakheri B, Pirseyedi SM, Mehdinejad N and Farsi M 2014. Isolation and characterization of novel microsatellite markers in walnut (Juglans regia L.). Amer. J. Plant Sci. 5: 409-415.

Perrier X and Jacquemoud-Collet JP 2006. DARwin software. http://www. darwin.cirad.fr/darwin.

Pradhan A, Yan G and Plummer JA 2004. Development of DNA fingerprinting keys for the identification of radish cultivars. Aus. J. Exp. Agr. 44: 95-102.

Pritchard JK, Stephens M and Donnelly P 2000. Inference of population structure using multilocus genotype data. Genet. 155: 945-959.

Shah UN, Mir JI, Ahmed N and Fazili KM 2016. Assessment of germplasm diversity and genetic relationships among walnut (Juglans regia L.) genotypes through microsatellite markers. J. Saudi Soc. Agri. Sci. http://dx.doi.org/10.1016/j. jssas. 2016. 07.005.

Victory ER, Glaubitz JC, Rhodes OE and Woeste KE 2006. Genetic homogeneity in Juglans nigra at nuclear microsatellites. Am. J. Bot. 93: 118-126.

Wang ZJ, Huang JG, Chuari J, Qin W and Hua F 2007. RAPD analysis on genetic diversity of Carya dabieshanensis populations. J. Plant Eco. 30: 534-538.

(Manuscript received on 7 February, 2019; revised on 19 March, 2020) 